\title{
Aportes de la didáctica de la historia del derecho a la cultura jurídica: formación de un sujeto histórico amoroso*
} The Teaching of the History of Law
and Legal Culture: Formation of a Loving
Historical Subject

\author{
Eric Eduardo Palma González ${ }^{* *}$ \\ María Francisca Elgueta Rosas***
}

\begin{abstract}
RESUMEN
Este artículo postula una didáctica de la historia del derecho centrada en la enseñanza-aprendizaje. Esta propone formar profesionales del derecho que promuevan la democracia, los derechos humanos y la interculturalidad. Con ello se da soporte a una nueva cultura juridica. Dicha orientación formativa está inspirada en el modelo pedagógico sociocrítico; por tanto, la cátedra se aleja del modelo de competencias para contribuir con la formación de un abogado como sujeto histórico amoroso.
\end{abstract}

PALABRAS CLAVE: Didáctica de la historia del derecho, enseñanza-aprendizaje del derecho, sujeto histórico, sujeto amoroso, cultura jurídica.

\begin{abstract}
This article suggests that the instruction of the History of Law Lecture be centered on teaching-learning principle. This proposes to train Legal Professionals that promote Democracy, Human Rights and Interculturality. With this thinking, a new Legal Culture is supported. This education orientation is inspired by the Socio-Critical Pedagogical Model; therefore, the Lecture moves away from the Model of Competencies to contribute to the development of a Lawyer as a Historical Subject of Love.
\end{abstract}

KEY WORDS: Didactics of the history of law, teaching-learning of law, historical subject, love subject, legal culture.

\footnotetext{
* Artículo de investigación. Recibido el 25 de julio de 2017 y aceptado para su publicación el 21 de mayo de 2018.

** Profesor en la Facultad de Derecho de la Universidad de Chile. (epalmag@derecho.uchile.cl) orcid.org/00000002-8804-6278

*** Profesora en la Facultad de Derecho de la Universidad de Chile. (cpudd2@derecho.uchile.cl) orcid.org/00000002-4212-3960
} 


\section{SUMARIO}

1. Introducción

2. El desafio de Fernando de Arvizu y Galarraga

3. Formación de la conciencia crítica o del sujeto histórico y amoroso

4. La zona oscura de la tradición jurídica occidental

5. Enseñanza del derecho precolombino, pluralismo jurídico e interculturalidad

6. Conclusiones

\section{Introducción}

La expresión historia del derecho es polisémica. No tiene solo un significado, sino varios que remiten a distintos planos de análisis del quehacer histórico jurídico. La historia del derecho se puede analizar como cátedra -enseñanzaaprendizaje-; investigación -método historiográfico- y en relación con su objeto de estudio -acontecer histórico jurídico-. Esta distinción es tan útil que permite describir y distinguir las concepciones histórico-jurídicas que se han manifestado a lo largo del tiempo. De este modo, se pueden caracterizar a partir de la posición que adopten respecto de estas tres dimensiones fundamentales. ${ }^{1}$

Estudiar la cátedra implica ocuparse de a) su historia y su posición en la malla curricular de la carrera; $b$ ) los programas de estudio; c) quiénes enseñaron; d) quiénes estudiaron; e) los textos de estudio; f) la organización de la facultad o escuela en que se imparte, y g) el método de enseñanza o de la didáctica, así como la evaluación.

Considerando la actualidad e importancia de las cuestiones relativas a la enseñanza del derecho ${ }^{2}$-temática de la que uno de nosotros se viene ocupando desde hace décadas ${ }^{3}$-, en este artículo, pretendemos seguir avanzando en la comprensión del modelo pedagógico y la didáctica que la inspira y caracteriza. Nuestro propósito es identificar la tendencia que existe sobre la enseñanza de la historia del derecho. Asimismo, buscamos proponer una didáctica que sea un soporte eficiente para la difusión y valoración de la cultura jurídica de los derechos humanos, del sistema democrático y la interculturalidad.

\footnotetext{
1 Palma, ERic, "Pasado, presente y futuro de la Historia del Derecho en Chile", Forum Historiae luris, 2009.

2 Desde el año 2005 se celebra en España el Congreso Nacional de docencia en Ciencias Jurídicas. Sus ponencias se publican como Actas.

3 Palma, Eric, "Pasado, presente y futuro de la Historia del Derecho en Chile", Forum Historiae luris, 2009.
} 
Recurriendo al método histórico y bibliográfico, y a los marcos teóricos que aporta la perspectiva pedagógica sociocrítica de Paulo Freire, pretendemos alcanzar los siguientes objetivos. En primer lugar, hemos de revisar la reflexión de la disciplina histórico jurídica sobre la enseñanza de la historia del derecho en las últimas tres décadas. Después, caracterizaremos someramente dicha reflexión e identificaremos el modelo pedagógico predominante en el tiempo presente. Después, tenemos como objetivo proponer una didáctica del derecho centrada en el aprendizaje del estudiante y cuyo objeto sea la formación de "conciencia crítica". Para ello, introduciremos las nociones de "sujeto histórico", "sujeto amoroso" y "sujeto histórico amoroso". Relacionaremos dicha conciencia crítica y el proceso de formación del sujeto histórico amoroso, con la denuncia de lo que llamamos "zona oscura de la tradición jurídica occidental". A partir de dicha visión crítica, hemos de formular una propuesta didáctica que aporte elementos para una enseñanza de la historia del derecho. Esto con el fin de enfrentar los desafíos de la construcción de una sociedad de pluralismo jurídico e interculturalidad.

Nuestra hipótesis es que, en la cátedra de Historia del Derecho, se puede realizar una práctica docente centrada en el aprendizaje del estudiante y que ésta ayude al surgimiento de su "conciencia crítica". Nuestras fuentes históricas son artículos sobre enseñanza de la historia del derecho y educación jurídica, que fueron publicados entre la década de 1980 y 2010. Estos artículos aparecen en las revistas de historia del derecho de España, México, Argentina y Chile, países donde la disciplina de historia del derecho goza de mayor robustez. Analizamos también los trabajos publicados en la misma época en las revistas de pedagogía jurídica de habla hispana.

Revisamos la reflexión sobre formación jurídica sin ningún ánimo de exhaustividad, pues sólo nos interesó establecer una tendencia, para encontrar qué domina los trabajos sobre enseñanza del derecho, la formación basada en competencias. Paralelamente, analizamos la presencia de temas de enseñanza en las revistas especializadas de historia del derecho, entre los años 1988 y 2016. Utilizamos como punto de partida un artículo del profesor español Fernando de Arvizu, publicado en 1988 en el Anuario de Historia del Derecho Español, ${ }^{4}$ porque en dicho trabajo el autor desafió a los iushistoriadores a ocuparse de este tema con sentido de urgencia.

Teniendo presente el escaso eco de aquel desafío, respondemos al reto considerando las peculiaridades del siglo xxi. Esto es, fundados en un

\footnotetext{
${ }^{4}$ Arvizu y Galarraga, Fernando de, "La enseñanza de historia del Derecho: reflexiones en busca de una polémica", Anuario de Historia del Derecho Español, 1988, pp. 491-498.
} 
modelo pedagógico y una didáctica sociocrítica, lo cual implica no recurrir al enfoque pedagógico dominante de la formación basada en competencias. Proponemos un proceso formativo en la cátedra de Historia del Derecho que denuncie lo que llamamos "zona oscura de la tradición jurídica occidental" y anuncie la nueva cultura jurídica de los derechos humanos, la democracia y la sociedad intercultural.

El artículo innova, pues propone que la enseñanza-aprendizaje de la historia del derecho debe enfocarse en la formación de un "sujeto histórico amoroso". Para ello, se centra en el aprendizaje del estudiante, en aras de la superación de la zona oscura de la tradición jurídica occidental. Esta tarea es ineludible para la cátedra, si pretende ser una contribución a la formación del jurista iberoamericano del siglo xxI, cuyo desafío más importante es contribuir a la construcción de una sociedad democrática, de derechos humanos y pluralismo jurídico.

Según se establece en numerosos trabajos publicados en la última década en las revistas especializadas, ${ }^{5}$ existe un importante vínculo entre formación basada en competencias y aprendizaje. Nuestro planteamiento configura una perspectiva distinta, al relacionar aprendizaje y formación sociocrítica según como lo entienden Palma y Elgueta. ${ }^{6}$

\section{El desafío de Fernando de Arvizu y Galarraga}

A propósito de su experiencia en España evaluando las oposiciones presentadas para acceder a una plaza de profesor funcionario en la cátedra de historia del derecho, en 1988, Fernando de Arvizu y Galarraga propuso una explicación sobre el desinterés respecto a los métodos de enseñanza de la disciplina.

Antes bien, lo que importaba y sigue importando, es la investigación que había realizado el candidato. Y esto pone sobre el tapete algo que es unánimemente sentido en todas las universidades de nuestra civilización occidental: es mucho más fácil evaluar la investigación que la docencia, y, por ello, se renuncia a la segunda, menospreciándola injustamente. ${ }^{7}$

\footnotetext{
${ }^{5}$ Véase las revistas de pedagogia jurídica Academia, Revista de Pedagogía Universitaria y Didáctica del Derecho, Educación y Derecho, Docencia y Derecho, Revista para la docencia jurídica universitaria y Revista Jurídica de Investigación e Innovación Educativa.

${ }^{6}$ Palma, Eric y Elgueta, Maria Francisca, "Enseñanza de la historia del derecho centrada en el aprendizaje de los estudiantes a lo largo de 115 años de la fundación de la cátedra (Chile, 1902)", Precedente. Revista Jurídica, núm. 12.

7 Arvizu y GalarRaga, Fernando de, "La enseñanza de historia del Derecho: reflexiones en busca de una polémica", Anuario de Historia del Derecho Español, 1988, p. 494.
} 
Desafió entonces a la disciplina y a los autores a superar tal deficiencia, y por ello nos ha servido como hito de este trabajo. ¿Qué ocurrió con la temática a partir de 1988? La revisión de las principales revistas de historia del derecho, a la luz del diagnóstico y propuesta referidos, configura un panorama desolador. La Revista Chilena de Historia del Derecho no tiene, en la etapa 19892014 -último número publicado-, ningún artículo cuyo tema central sea la enseñanza y didáctica de la historia del derecho.

La Revista de Estudios Histórico Jurídicos, también chilena, presenta un panorama semejante, sin embargo, hay algunas noticias sobre la evolución de la historiografía y la cátedra. Han publicado en la revista las autoras españolas Patricia Zambrana y María Encarnación Gómez Rojo, que, según veremos, pueden ser identificadas como las que vienen trabajando en España con mayor regularidad sobre historia de la cátedra, historiografía comparada y enseñanza de la historia del derecho.

La revista argentina, Revista de Historia del Derecho, publicada por el Instituto de Investigaciones de Historia del Derecho, tuvo entre los años de 1994 y 1999 una sección titulada "Enseñanza de la Historia del Derecho". Ahí, se publicó, en 1997, "El contenido de la Historia del Derecho según el nivel en que se enseña”, de María Rosa Pugliese Lavalle, y "La enseñanza de la Historia Jurídica en el ámbito de los historiadores (reflexiones y propuesta)", de María Cristina Seghesso de López Aragón. En 1998, se publicó el trabajo de Ezequiel Abásolo, "La enseñanza de la Historia del Derecho y una polémica entre Ricardo Levene y Jorge Cabral Texo”; en 1999 el trabajo de María Isabel Seoane, "Los recursos didácticos a utilizar en la enseñanza de la Historia del Derecho Argentino"; y, en el 2011, una reseña de la obra de Pio Caroni sobre la enseñanza histórico-jurídica por Rosa María Pugliese.

La Revista de Historia del Derecho Ricardo Levene, en el periodo 1989-1998, publicó el importante trabajo de Abelardo Levaggi, "Consideraciones sobre la enseñanza de la Historia del Derecho". ${ }^{8}$ Según veremos más adelante, puede que éste sea el aporte más significativo de todo el periodo analizado.

Por su parte, el Anuario Mexicano de Historia del Derecho, fundado en 1989, y denominado a partir de 2011 Revista Mexicana de Historia del Derecho, no contiene ningún trabajo entre los años de 1989 y 2016.

Para el caso español, la revista española Ius fugit: Revista interdisciplinar de estudios histórico-jurídicos, desde su fecha de fundación, en el año 1992,

${ }^{8}$ Levaggi, Abelardo, "Consideraciones sobre la enseñanza de la Historia del Derecho", Revista de Historia del Derecho Ricardo Levene, vol. 27. 
hasta el año 2016, no contiene trabajos específicos, sin embargo, hay relevantes artículos sobre universidades y universitarios.

En la revista española Cuadernos de Historia del Derecho, entre los años de 1994, fecha de su primer número, y el año 2016, Antonio Pedro Barbas Homem y por Amelia Gascón Cervantes publicaron, en 2009, "Dos reflexiones sobre la actual enseñanza del Derecho”, referidos a la situación de Portugal y México.

En el Anuario de Historia del Derecho Español (AHDE) encontramos que, en 1988, se publicó el artículo referido de Fernando de Arvizu y Galarraga sobre la enseñanza de la disciplina y sus defectos; en 1996, Max Turrull dio noticia de la manualística y programas de historia del derecho en Francia -bajo el apartado historiografía, no el de enseñanza del derecho-. En el año 2000, Patricia Zambrana dio un breve vistazo a la enseñanza histórico-jurídica en algunas universidades de Estados Unidos de América y Canadá. Lo mismo hizo en el 2002, a propósito del caso italiano, y en el 2006, respecto de Brasil. Este mismo año, María Gómez y Belén Malavé escribieron sobre el estado de la enseñanza en Austria, el trabajo fue publicado en la sección "Varia".

En síntesis, aunque las publicaciones son escasas, destacan por su interés en la materia los casos argentino y español; por su desinterés, el mexicano y el chileno. ${ }^{10}$ Desde 1988, momento del desafío y crítica de Arvizu, al 2016, la enseñanza y la didáctica de la historia del derecho tienen un lugar muy menor en las principales revistas de la especialidad publicadas en Chile, Argentina, México y España. Los artículos inciden en alguno de los temas que ya identificamos como propios de una historia de la cátedra, sin agotarlos y sin mayor profundidad, siendo muy escasa la atención a la didáctica como tal.

Sin embargo, en España, el impacto del Plan Bolonia de formación del espacio europeo de educación superior ha provocado que algunos historiadores del derecho se ocupen de introducir en la enseñanza de la historia del derecho, bajo el modelo de formación por competencias, las nuevas ideas sobre el aprendizaje y también el uso de las tic. Sus perspectivas se vinculan a un modelo técnico al servicio de la profesionalización, que poca relación tiene con la propuesta sostenida en este artículo.

La voz cátedra presenta un mayor alcance que la expresión enseñanza o didáctica, empleada por Birocchi y Caroni, a propósito de sus reflexiones

\footnotetext{
${ }^{9}$ Cabe destacar el trabajo de María Paz Alonso Romero, que da noticia, en la sección documentos, de algunos esfuerzos realizados en el siglo xvı para abarcar teoría y práctica en la enseñanza. Asimismo, es relevante el de Martínez Neira de 1998, también en sección documentos, sobre la enseñanza del derecho bajo Carlos IV.

${ }^{10}$ El propósito de este trabajo no es dar cuenta de todo lo publicado. Por lo tanto, no sería correcto concluir que el desinterés mostrado en las revistas implique una falta total de reflexión. De hecho, por mero ejemplo, en 1954, el chileno Anibal Bascuñán publicó su libro Pedagogía Jurídica, donde aborda algunas cuestiones relativas a la enseñanza histórico-juridicas. Eric Eduardo Palma viene publicando desde 1995 trabajos que abordan la temática.
} 
acerca de la posición y el valor de la historia del derecho en la malla curricular de derecho. ${ }^{11}$ Las ideas aquí expuestas, desarrolladas con autonomía de las ideas de Caroni, aunque en tiempos más o menos semejantes, relacionan las tres dimensiones de la historia del derecho para dar un sentido distinto a la expresión didáctica, enseñanza y formación crítica.

En efecto, vamos a ocuparnos del aprendizaje desde una perspectiva distinta a la usual, que está centrada en las competencias. ${ }^{12}$ Así, nos proponemos orientar la formación hacia la emergencia de una conciencia crítica.

\section{Formación de la conciencia crítica o del sujeto histórico y amoroso}

A propósito de la historia del derecho, es factible desarrollar un programa, es decir, contenidos, así como un tipo de investigación y una práctica docente que ayude no sólo a la formación de una "actitud crítica", sino al surgimiento de una conciencia crítica, tanto en el profesor como en el alumno. El profesor de historia del derecho, interesado en hacer del acto educativo una instancia de formación de la conciencia crítica, ayuda a que el estudiante se transforme en sujeto histórico.

Esta transformación significa que el estudiante a) es un individuo consciente de sí mismo y de su valor intrínseco en tanto que individuo único; b) es consciente de su condición de ser social y, por ende, conocedor de las peculiaridades del tiempo histórico que le toca vivir y de su contexto social inmediato; c) es capaz de entender cómo las fuerzas sociales, políticas, económicas y culturales determinan las características de la sociedad en la que vive $\mathrm{y}$, por lo tanto, del derecho que la rige; $d$ ) está interesado en denunciar el modelo tradicional de organización y en anunciar un nuevo modelo; e) está interesado en actuar para la transformación. ${ }^{13}$

Según nuestra propuesta, este sujeto histórico debe ser, al mismo tiempo, un sujeto amoroso. Es decir, el sujeto debe estar convencido del valor de la vida en comunidad; comprometido con el anuncio y promoción activa de la cultura de derechos humanos (cultura humanitaria); comprometido con la promoción de relaciones humanas en que el "otro", el distinto, el ajeno, se vivencie

\footnotetext{
${ }^{11}$ Caroni, Pio, La Soledad del Historiador del Derecho. Apuntes sobre la conveniencia de una disciplina diferente, Madrid, Universidad Carlos III de Madrid, 2010.

${ }^{12}$ Véase por todos la Revista Juridica de Investigación e Innovación Educativa (REJIE) (2010-2017), de la Facultad de Derecho Universidad de Málaga, en la que destacan los trabajos sobre enseñanza de la historia del derecho y del derecho romano.

${ }^{13}$ Palma GonzÁlez, Eric, "Pasado, presente y futuro de la Historia del Derecho en Chile", Revista electrónica alemana Forum Historiae luris.
} 
como legítimo otro; y una persona consciente de sus hábitos -normalmente patriarcales-, y en su caso, de la necesidad de su transformación. ${ }^{14}$

Un sujeto histórico comprende su situación personal en el presente, pues es capaz de entender su individualidad como producto del devenir social y, para ello, no es suficiente memorizar palabras, fechas, nombres. Es necesario que comprenda cómo distintos factores -económicos, políticos, relativos a la organización social y vinculados a ciertos intereses y valores- han venido moldeando la sociedad en la que vive, así como otros instrumentos de control social, a través del derecho.

La actitud crítica y la conciencia crítica tienen un sentido distinto. Mientras la formación de la actitud se traduce en el desarrollo de la capacidad de análisis, síntesis y evaluación, la segunda implica una comprensión y apropiación de la realidad para su transformación, en miras a la construcción de una sociedad que respete la libertad y la dignidad humana. Mientras la primera forma un profesional crítico, la segunda, un sujeto histórico amoroso. Mientras la primera atiende a la eficacia profesional y la productividad, la segunda, a la justicia y el compromiso social con la democracia y los derechos humanos, en el ejercicio de la profesión.

¿Qué práctica docente contribuye a nuestro juicio y, según nuestra propuesta, a la formación de un sujeto histórico amoroso? La que abandona la narración de hechos históricos como meros datos y procura una apropiación de la historia como sucesos vividos por gente de carne y hueso, cuyas vidas fueron objeto de regulación de determinadas fuerzas sociales, en función de ciertos valores e intereses.

Una cátedra así enfocada debe procurar que los estudiantes alcancen progresivamente los siguientes objetivos de aprendizaje: conocer, describir, generar, analizar, relacionar y aplicar la información histórico-jurídica. Cada uno de estos objetivos se puede relacionar con tres dimensiones y sus respectivas habilidades: dimensión cognitiva, dimensión procedimental y dimensión político-normativa. En el plano cognitivo, la didáctica busca el desarrollo de la capacidad de:

\footnotetext{
${ }^{14}$ La noción pensamiento critico suele emplearse en la docencia universitaria sin un alcance claro, incluso. Gustavo Hawes señala que "suele confundirse 'pensamiento crítico' con acepciones que están más o menos lejanas de su concepto: aprendizaje activo, taxonomía de Bloom, aprendizaje colaborativo, inteligencias múltiples, herramientas como mapas conceptuales". El autor distingue pensamiento crítico como producto, como práctica y siguiendo a Habermas, como praxis (siendo este último el vinculado a la noción de sujeto histórico amoroso construida a partir de Freire y Maturana-Varela). Hawes, Gustavo, Pensamiento crítico en la formación universitaria. Documento de Trabajo 2003/6 Proyecto Mecesup TAL 0101, Universidad de Talca, 2003.
} 
- Dar noticia de la información histórico-jurídica

- Reconocer conceptos histórico-jurídicos

- Identificar relaciones entre conceptos histórico-jurídicos

- Identificar teorías histórico-jurídicas

- Explicar fenómenos de la realidad histórico-jurídica, utilizando conceptos y teorías histórico-jurídicas

- Utilizar conceptos y teorías histórico-jurídicas para dar noticia de la realidad local, provincial, nacional y mundial del siglo XXI

- Identificar, a lo largo de la historia, relaciones entre el medio social y el fenómeno normativo jurídico

- Explicar el derecho como producto social

- Explicar la influencia del derecho en la configuración de ciertas características del medio social

En la dimensión procedimental, se busca:

- Generar la habilidad para buscar, procesar y analizar información

- Desarrollar la capacidad de redactar artículos científicos

- Desarrollar la capacidad de exponer oralmente síntesis de ideas ajenas y propias

- Desarrollo de la habilidad para formar y hacer funcionar equipos de trabajo

Finalmente, en el plano político-normativo, el profesor iushistoriador debe inspirar en sus estudiantes un compromiso abierto con la honestidad; la responsabilidad en el trabajo académico; el respeto a la diversidad de toda índole; el desarrollo del pensamiento sociocrítico, así como un alejamiento consciente de los hábitos patriarcales de organización. Debe alimentar una mirada también crítica de la tradición jurídica occidental y la valoración de la democracia y la cultura humanitaria o de los derechos humanos - construida a partir de las declaraciones de derechos que se inician con la Declaración Universal de Derechos Humanos de 1948-.

Esta propuesta educativa, según la cual el educando es un agente de transformación que se comporta como sujeto histórico amoroso, tiene claro fundamento normativo jurídico. Éste es el Pacto Internacional de Derechos Económicos, Sociales y Culturales (PIDESC), de 1976. Sus artículos 13 y 15 disponen, entre otras cosas, la libertad para la investigación científica y

que la educación debe orientarse hacia el pleno desarrollo de la personalidad humana y del sentido de su dignidad, y debe fortalecer el 
respeto por los derechos humanos y las libertades fundamentales. Convienen asimismo en que la educación debe capacitar a todas las personas para participar efectivamente en una sociedad libre, favorecer la comprensión, la tolerancia y la amistad entre todas las naciones y entre todos los grupos raciales, étnicos o religiosos.

La Ley 20.370 (Ley General de Enseñanza, 2009), en su artículo 2, señala que en Chile, país desde el que generamos esta reflexión: "La educación [...] Se enmarca en el respeto y valoración de los derechos humanos y de las libertades fundamentales, de la diversidad multicultural y de la paz, y de nuestra identidad nacional, capacitando a las personas para conducir su vida en forma plena, para convivir y participar en forma responsable, tolerante, solidaria, democrática y activa en la comunidad, y para trabajar y contribuir al desarrollo del país".

También se encuentra el DFL 1 Ministerio de Educación, del 10 de marzo de 2006, que establece el Estatuto de la Universidad de Chile. En su artículo 1 quáter, éste dispone:

Los principios inspiradores que guían a la Universidad en el cumplimiento de su misión inspiran la actividad académica y fundamentan la pertenencia de sus miembros a la vida universitaria, son: la libertad de pensamiento y de expresión; el pluralismo; y la participación de sus miembros en la vida institucional, con resguardo de las jerarquías inherentes al quehacer universitario. Forman parte también de estos principios orientadores: la actitud reflexiva, dialogante y crítica en el ejercicio de las tareas intelectuales; la equidad y la valoración del mérito en el ingreso a la Institución, en su promoción y egreso; la formación de personas con sentido ético, cívico y de solidaridad social; el respeto a personas y bienes.

278 Cabe considerar también la posición de la unEsco en lo tocante a la libertad académica, en el contexto de una sociedad democrática:

VI. Derechos y libertades del personal docente de la enseñanza superior. 26. Al igual que todos los demás grupos e individuos, el personal docente de la enseñanza superior debe gozar de los derechos civiles, políticos, sociales y culturales reconocidos internacionalmente y aplicables a todos los ciudadanos. En consecuencia, todo el personal docente de la enseñanza superior debe disfrutar de la libertad de pen- 
samiento, conciencia, religión, expresión, reunión y asociación, así como del derecho a la libertad y seguridad de la persona y la libertad de movimiento. ${ }^{15}$

Para alcanzar su objetivo formativo, la investigación y la cátedra deben asumir una tarea de denuncia de los obstáculos que han impedido configurar sociedades plenamente respetuosas del ser humano, orientadas a la promoción de la participación política y el respeto a la integridad física y psíquica de los mismos. Uno de esos obstáculos es el peso, en el devenir de las sociedades, de hábitos contrarios que han tenido protección jurídica y, por ende, se les ha dado un lugar legítimo en el mundo del derecho.

¿Qué didáctica se requiere para contribuir a dicho objetivo formativo? Un ejemplo concreto permite observar el tipo de didáctica que puede colaborar al desarrollo de las tres dimensiones de aprendizaje ya descritas. A propósito de la historia del derecho indiano (derecho colonial español) realizamos con los estudiantes una evaluación que se extiende a lo largo del semestre, relativa a la polémica de Indias o la querella de los Justos Títulos. Antes de iniciar el trabajo, realizamos un taller de alfabetización informacional para que conocieran las técnicas de búsqueda de información especializada en la web (fuentes históricas y bibliográficas) y un taller de presentación informes. El trabajo respecto de la polémica comprende distintas fases. ${ }^{16}$

La primera fase fue de motivación para con el tema. En ésta, el profesor realiza una exposición acerca del debate jurídico teológico que generó la cuestión del tratamiento del indio americano, y la relevancia del mismo para los debates del tiempo presente, en que la interculturalidad y la plurinacionalidad buscan un espacio en el derecho interno de los países iberoamericanos. Para situar a los alumnos en algunos elementos centrales de la materia, y reforzar la motivación, se les exhibe la película La Controversia de Valladolid.

En la siguiente fase, se realiza la presentación del tema. Se inicia con la lectura por parte de los alumnos, fuera del salón de clases, de un artículo científico relativo a la polémica de Indias. Dicho material les permite identificar las aristas de la temática.

La tercera fase es de representación. A partir de la motivación y la presentación, los estudiantes deben verificar por sí mismos el grado de entendimiento del tema. El artículo de la presentación debe permitirles identificar las pos-

\footnotetext{
${ }^{15}$ UNESCO, "Recopilación de recomendaciones relativas a la situación del personal docente de los años 1966 y 1997 ", UNESCO. Disponible en: http://unesdoc.unesco.org/images/0016/001604/160495s.pdf

${ }^{16}$ Esta propuesta de planificación se desarrolla en el Programa de Diplomado en Docencia Universitaria de la Universidad Metropolitana de Ciencia de la Educación, donde la conocimos en el año 2004.
} 
turas contrapuestas para hablar de una polémica o controversia. Enseguida, realizan una búsqueda autónoma e individual de fuentes históricas y bibliografía especializada que avale las posturas identificadas, debiendo entregar un informe al respecto.

La siguiente fase es de cuestionamiento. Luego de la entrega de los informes, se constituyen equipos de trabajo y se realiza un sorteo para determinar por azar a qué grupos les corresponde la defensa de las ideas y planteamientos de Juan Ginés de Sepúlveda o de fray Bartolomé de las Casas. Puede ocurrir, y de hecho ocurre, que a los estudiantes les toque defender una posición que no comparten. Verbigracia, simpatizando con Sepúlveda deberán defender las ideas de Las Casas.

Hecha la elección, cada grupo debe preparar un nuevo informe, esta vez, sosteniendo la postura que han sorteado. Ello exige identificar con claridad las ideas y posturas por defender y contar con un adecuado apoyo de fuentes históricas y estudios especializados. Dicho informe se entrega para ser puesto en conocimiento de la otra parte.

La última fase es la del Desempeño. Finalmente, se cita a un día y hora determinado a dos grupos con posturas contrapuestas para que confronten, de manera oral, y sin poder leer un texto escrito de respaldo, sus respectivas alegaciones. Todos los integrantes del grupo deben intervenir en los alegatos, ya sea como orador principal o secundario. Escuchadas las partes y sus alegaciones principales, el profesor identifica los temas controvertidos y las colisiones de los argumentos. Corresponde a los oradores que hacen uso de la palabra en el segundo momento hacerse cargo de los argumentos que cuestionan las bases de sus alegaciones, y que les son presentados por el profesor como colisiones de argumentos.

Cada fase de este proceso, el cual se extiende a lo largo del semestre, implica el desarrollo de una o más de las habilidades identificadas como dimensiones cognitivas, procedimentales y político normativas. Ocurre que, en el transcurso del tiempo, van desarrollando la lectura y escritura situada, dado que se familiarizan con textos jurídicos y redactan géneros propios del derecho.

Los alumnos aprenden a trabajar en equipo. Puesto que se trata de un ejercicio para el primer año de la carrera, los alegatos pueden transformarse en su primera práctica de oralidad y oratoria, a propósito de una cuestión eminentemente jurídica. Esto aunado a que tienen una primera experiencia en investigación, entrando en contacto con fuentes históricas y bibliografía especializada. De este modo, los estudiantes se ven inmersos en un problema jurídico, moral y político de enorme trascendencia histórica con proyección al tiempo presente.

Con el fin de comprender el alcance del debate, los estudiantes deben pasar de los textos legales al conocimiento de la realidad social, política y económica. 
Es decir, deben comprender el contexto de producción social del derecho, identificando las motivaciones -valores e intereses- de la Corona, de la Iglesia, de los vecinos y de las propias comunidades indígenas. Comprenderán que la reflexión sobre la "naturaleza" del indígena americano, y el lugar que se le adjudicó como incapaz relativo, tuvo como base una mirada europea que se construyó determinada por múltiples factores. Varios de ellos siguen operando en la sociedad actual. Nuevamente, se debate sobre el lugar de las comunidades indígenas en nuestras sociedades.

Este ejercicio implica también la superación de un cierto tipo de clase magistral, que viene siendo criticada desde hace muchas décadas. Esta es la meramente expositiva. ${ }^{17} \mathrm{Al}$ respecto, en 1990, el maestro Abelardo Levaggi sostuvo, en un trabajo cuyo objeto principal fue el tema pedagógico, la necesidad de superar los defectos de esta cátedra

Atendiendo sólo a las ventajas que tiene la clase magistral no se suele reparar en sus inconvenientes para los estudiantes, quienes se convierten en objeto, pasivo, de la clase, sin la posibilidad de participar en ella, de ejercitar su inteligencia, y de beneficiarse con una enseñanza más integral y creativa. Cuanto mayor sea el interés que se suscite en el estudiante, cuanto más agradable le sea la clase, cuanto mayor sea su participación en ella, mejor será el resultado [...] Para eso, además de tener idoneidad científica, [el profesor] debe estar convencido de la importancia que tiene el grupo dinámico como motivador de voluntades; ser capaz de estructurar su trabajo, y de crear un clima distendido de confianza y respeto mutuos; de mantener el equilibrio entre todos los miembros, estimular la participación de los renuentes y moderar los excesos; de escuchar a los demás y de reservar para el momento oportuno la manifestación de sus ideales. Las técnicas que puede emplear son varias. Considero que se adaptan bien a la enseñanza de esta materia el análisis grupal de textos, la representación combinada con el "fórum", y el simposio también combinado con el "forum". ${ }^{18}$

\footnotetext{
${ }_{17}$ Para una propuesta sobre tipos y utilidad de la clase magistral, véase de Elgueta Rosas, Maria Francisca y Palma, Eric EduARDO, "Una propuesta de clasificación de la clase magistral impartida en Derecho", Revista Chilena de Derecho, núm. 2014.

${ }^{18}$ Levaggl, Abelardo, "Consideraciones sobre la enseñanza de la Historia del Derecho", Revista de Historia del Derecho Ricardo Levene, núm. 27, pp. 162-163. Arvizu y galarraga (1988, 496-497) sostuvo a este respecto: "Si bien es cierto que el profesor no debe buscar el halago del auditorio, no lo es menos que no puede encerrarse en una torre de marfil, y dedicarse a explicar lo que le plazca, menospreciando no solo lo que pueda tener interés para el alumnado dentro de la asignatura, sino el propio momento histórico en el que desarrolla su curso. Pero si mantengo que una actitud docente desconectada de intereses del alumnado, además de no ser cientificamente aceptable, conduce -ya se ve- al menosprecio y al aislamiento de la disciplina y de quienes la cultivan".
} 
El programa de estudios de la cátedra se nutre con la investigación históricojurídica, por lo tanto, la determinación del objeto de estudio, la metodología de investigación, el proceso investigador, así como los fines que se asignan a la investigación, contribuyen al desarrollo de una docencia reproductora o libertadora. ${ }^{19}$

[Esta última] implica un acto permanente de desvelamiento de la realidad [...] busca la emersión de las conciencias, de la que resulta su inserción crítica en la realidad. Cuanto más se problematizan los educandos, como seres en el mundo y con el mundo, se sentirán mayormente desafiados [...] La educación como práctica de la libertad, al contrario de aquella que es práctica de la dominación, implica la negación del hombre abstracto, suelto, aislado, desligado del mundo, así como la negación del mundo como una realidad ausente de los hombres. ${ }^{20}$

\section{La zona oscura de la tradición jurídica occidental}

La creación de conocimiento histórico, es decir la actividad historiográfica, se ha hecho, como viene afirmando Palma, sin atender a la memoria de los sujetos que han sufrido la violencia, la dominación y la discriminación, y a propósito de cuya vida cotidiana se configuró una jerarquía social. La vida social de Occidente puede caracterizarse como la recreación milenaria de un conjunto de hábitos a los que englobamos bajo la denominación de "mentalidad patriarcal, y que implican la legitimación de conductas sociales que aceptan y promueven como normales en las relaciones entre las personas la violencia, la discriminación, la dominación, $y$, como resultado, una jerarquía social". ${ }^{21}$

La narración histórica de los textos y del aula no realiza una operación de ocultamiento de dicho fenómeno, sino de justificación y de legitimación, en determinados casos de la violencia y sus derivados. 0 bien, bajo argumento de neutralidad y objetividad, guarda silencio sobre sus causas y formas de manifestación, como si la única tarea admisible fuera limitarse a darla a conocer.

La violencia se ejerce normalmente respecto de marginales, es decir, perdedores o inferiores; sobre enemigos; o sobre seres cuyo valor es tan insignificante que no amerita dejar testimonio del fenómeno. El problema de

\footnotetext{
${ }^{19}$ Freire, Paulo, Pedagogía del Oprimido, México, Siglo XXI, 2007, p. 75. En América Latina, Enrique Dussel ha venido desarrollando en las últimas décadas la llamada filosofia de la liberación.

${ }^{20}$ Freire, Paulo, Pedagogía del Oprimido, México, Siglo XXI, 2007, p. 76.

${ }^{21}$ Palma González, Eric, "Pasado, presente y futuro de la Historia del Derecho en Chile", Revista electrónica alemana Forum Historiae luris.
} 
la descripción o de la narración no es la falta de visibilidad de la violencia; muy por el contrario, ella se presenta en ciertas historias acompañada de una estigmatización del vencido.

Los griegos tuvieron una imagen de su propia cultura como una de carácter superior, de manera tal que dieron pie para la distinción entre civilización y barbarie, entre civilizado y bárbaro, entre griego y extranjero. Lo propio aconteció con los romanos. ${ }^{22}$ Las relaciones con los extranjeros o bárbaros no siempre fueron pacíficas, por lo cual el extraño pasó a constituirse, en algunos momentos de la historia, en enemigo. No contentos con esta distinción, sostuvieron que, en la propia sociedad griega, o romana, era posible diferenciar a los individuos del cuerpo social: algunos degradaban su condición cayendo en la esclavitud. Así, entre los propios griegos, había criaturas superiores e inferiores.

A partir de esta diferenciación, se aceptó que algunos estaban llamados por su condición de superioridad a organizar la sociedad, y otros, por su naturaleza inferior, a implementar dicho modelo de organización que se les presentó como ideal por quienes ostentaban el poder.

En este sentido, es clarificadora la institución de la patria potestad. Según Guillermo Suarez:

La patria potestad romana es un poder jurídico viril [...] fue un poder jurídico (ex iure quiritium) y polivalente, que cumplía diversas finalidades: i) Personal, de naturaleza jurídica privada, pues se ejerce, sobre los hijos, en el estricto ámbito de la familia y de la domus; ii) Personal, de naturaleza jurídica pública, porque produce efectos jurídicos relacionados con la adquisición de la ciudadanía romana (vale decir nacionalidad) que repercuten en la vida y en la ordenación de colectividad; iii) Personal, de naturaleza jurisdiccional, porque el paterfamilias puede enjuiciar actos ilícitos penales y crímenes cometidos por los hijos; iv) Personal, de naturaleza coercitiva, porque el paterfamilias puede imponer penas a todos los sometidos; v) Personal, de naturaleza jurídica religiosa, porque habilita a la dirección de los cultos religiosos y de los dioses domésticos; vi) Personal, de naturaleza económica, absorbente, calificada de inhumana por Justiniano (quod nobis inhumanum visum est), pues todas las adquisiciones de los sometidos sub potestate pasan ipso iure al patrimonio del paterfamilias [...] el paterfamilias dirige y

${ }^{22}$ González Ballesteros, IVÁn, "El estereotipo del bárbaro y la imagen de la civilización en el occidente romano en la Geografía de Estrabón", Espacio, Tiempo y Forma, serie 2, tomo 22. 
gobierna la domus de la misma forma que los magistrados y el emperador gobiernan la República. ${ }^{23}$

La historia de la tradición jurídica occidental, a partir del siglo III antes de Cristo -época de inicio del expansionismo romano- puede ser vista también como la historia de la legitimación, justificación, promoción y defensa de este conjunto de hábitos que se presentó incluso como modelo de organización en todos los ámbitos: familiar, social, económico y político.

El derecho fue empleado para garantizar la proyección de estas conductas constitutivas de lo que llamamos hábitos o mentalidad patriarcal. Su expresión más significativa está en la regulación jurídica de la esclavitud. Una sociedad que admite la esclavitud, aunque la condene moralmente o la presente como institución contraria al derecho natural, configura como actos legítimos la violencia, la dominación, la discriminación sobre individuos con los que se convive cotidianamente. Esto sucede aún más si permite justificar una jerarquía social. Cabe recordar que el primer atributo del ateniense civilizado y del ciudadano romano era la libertad.

Estos hábitos patriarcales fueron justificados mediante doctrinas, por ejemplo, las de Aristóteles, que configuraron un entramado de relaciones sociales caracterizadas porque uno o más grupos sociales son objeto de dominación y discriminación, con lo cual se genera una jerarquía social.

Como estos fenómenos de atropello vienen legitimados por los hábitos y la doctrina, así como por el derecho, no hay espacio ni en la narración histórica ni en el aula para la configuración de una visión crítica de ellos. La mayor parte de la manualística histórico-jurídica española, mexicana, argentina y chilena, da noticia, sin reflexión crítica asociada, de los conceptos de bárbaro, vagabundo, ciudadano, esclavitud, guerra justa, patria potestad, potestad marital, incapacidad absoluta, súbdito, hereje, indígena, entre otros. Sin embargo, se omite una reflexión sobre los procesos de estigmatización que implican estas categorías sociales, por ende, no se denuncia el sufrimiento de ciertos grupos por parte del poder que se alza como superior, sino que sólo se constata su presencia. Para la historia oficial, pareciera que sólo existen como parias.

Dicho relato, que se presenta como neutralidad objetiva, tiene en el plano de la didáctica el inconveniente de trasladar la "aparente legitimidad" del pasado, al presente. Al hacerlo, se impide que aflore la conciencia crítica, porque se

${ }^{23}$ SuÁrez Blázouez, Guillermo, "La patria potestad en el derecho romano y en el derecho altomedieval visigodo", Revista de Estudios Histórico Juridicos, núm. 36, pp. 159-187. 
dan por válidos ciertos hábitos patriarcales con fuerte raigambre histórica que algunos consideran, todavía hoy, como legítimos.

De un tiempo a esta parte, como se refleja en la propuesta de Thomas Duve sobre una historia del derecho trasnacional, los historiadores del derecho europeo también han empezado a llamar la atención sobre esta dimensión:

volviendo a la remisión a Europa como el continente que produjo todos los logros culturales y jurídicos, tendríamos que preguntarnos si es realmente justo hacer un balance positivo, como solemos hacer habitualmente. ¿No proclamábamos la libertad y la igualdad en nuestros reinos, y practicábamos el racismo y la discriminación en otras partes de nuestros imperios? ¿No pagábamos nuestros éxitos culturales con lo que nos llevábamos de aquellos a quienes veíamos como "no civilizados”? ¿No ha sido la misma Europa de la libertad también el continente de los genocidios, las guerras mundiales y el colonialismo? ¿Podemos separar una cosa de la otra, las hazañas culturales de las crueldades increíbles? ${ }^{24}$

Cabe preguntarse, como hizo Palma, ¿cómo afecta a la vigencia del Estado de derecho que la educación de los abogados no incorpore también una mirada crítica de la tradición jurídica occidental? ¿Qué relevancia tiene este fenómeno para comprender la debilidad en la América lusohispana de la cultura humanitaria, es decir, la que promueve una convivencia fundada en la democracia y los derechos humanos? Necesitamos una historiografía jurídica y una enseñanza del derecho que se ponga al servicio de los marginados de la historia. En alguna manera, una historia de denuncia que dé cuenta de las operaciones de estigmatización que se realizan desde la élite con el propósito de justificar la violencia que domina, y, de paso, destruye la dignidad humana.

Para el caso de Chile, el programa de la cátedra de Historia del Derecho ha tenido a la historia del derecho español como contenido a lo largo del tiempo, con mayor énfasis a partir de la década de 1930. El conocimiento -estudio y enseñanza- del pasado jurídico español se justificó entonces, y se justifica mayoritariamente, hoy día, por la necesidad de comprender la historia del derecho chileno en el marco del derecho europeo. ${ }^{25}$

\footnotetext{
${ }^{24}$ Duve, Thomas, "Los desafios de la Historia Jurídica Europea", Anuario de Historia del Derecho Español, tomo 86, pp. 810-845.

${ }^{25}$ Véase Salvat Monguillot, Manuel, Breve historia del estudio del derecho, Chile, Ediciones del Instituto de Historia del Derecho Juan de Solórzano y Pereyra, 2001.
} 
Debido a la conquista española de América, se propone comprender el derecho nacional como una manifestación más de la denominada tradición jurídica occidental o derecho occidental, entendido como una familia jurídica. $\mathrm{Al}$ inicio de la expansión española, ya se había manifestado en la historia europea una parte significativa de los ordenamientos jurídicos que conforman el llamado derecho occidental. Es, precisamente, a través de España que participamos de esta experiencia. Así, el propósito del estudio del derecho español sería comprender cómo se configuró la tradición jurídica de la que formamos parte. Interesa conocer las raíces del derecho nacional que lo entroncan con el derecho occidental y la civilización católica occidental que lo creó.

Para el estudio de la tradición jurídica, se ha empleado la perspectiva, a estas alturas ya clásica, de John Henry Merryman. Según este autor, la tradición jurídica es:

Un conjunto de actitudes profundamente arraigadas y condicionadas históricamente acerca de la naturaleza de la ley, acerca de la función del derecho en la sociedad y en la forma de gobierno, acerca de la organización y operación apropiadas de un sistema jurídico y acerca del modo como el derecho debe crearse, aplicarse, estudiarse, perfeccionarse y enseñarse. La tradición jurídica relaciona el sistema jurídico con la cultura de la cual es una expresión parcial. Coloca al sistema jurídico dentro del ámbito cultural. ${ }^{26}$

Merryman explica lo que denomina tradición del derecho civil, que llama también "del derecho civil o escrito". Según la explicación del autor, se origina con la puesta en vigencia de las denominadas Doce Tablas (450 a. de C.) en Roma. Distingue en esta tradición subtradiciones: "derecho civil romano, derecho canónico, derecho mercantil, la revolución y la ciencia jurídica”. ${ }^{27}$

La adscripción del derecho patrio a la tradición del derecho civil escrito se ha estudiado como un caso de proyección del derecho español en América, así como de la cultura que lo sustenta, que se caracteriza como civilización católica occidental. La perspectiva de las últimas décadas ha significado que se destaque su vinculación con el derecho romano y el derecho canónico, según como se manifestó en el derecho común.

Respecto a esta tradición jurídica occidental españolizada -variante de la tradición del derecho civil escrito-, se despliega una visión positiva, incluso

\footnotetext{
${ }^{26}$ MerRyman, John Henry, La tradición Jurídica romano-canónica, México, FCE, 1971, p. 15.

${ }^{27}$ MerRYMan, John HenRY, La tradición Jurídica romano-canónica, México, FCE, 1971, p. 22.
} 
laudatoria, en la que se destaca el nivel científico del derecho y su componente moral -manifestación de la religión católica apostólica romana-. Su máxima expresión se encuentra, por lo mismo, en el derecho común, actualización de la obra de Justiniano. Al respecto, Duve señala:

El resultado es un panorama desproporcionado de "recepción del derecho europeo" por todo el mundo, que en ocasiones ha sido reafirmado por historiadores del derecho no europeos entusiasmados por descubrir y quizá incluso enfatizar los elementos europeos en sus propias tradiciones jurídicas, debido a la connotación positiva que esto daba a su propia historia en un tiempo en el que ser parte de la "cultura jurídica europea" se presentaba como formar parte de las "naciones civilizadas". ${ }^{28}$

La tradición jurídica occidental presenta el valor altamente positivo del desarrollo de los estudios de derecho, pero, tiene también responsabilidad en la proyección de la mentalidad patriarcal en el tiempo. A este respecto, Villacañas ha dicho: "Ciertos sistemas intelectuales ponen el mayor énfasis, las mejores energías, las mejores ideas y creencias, el mejor espíritu sistemático en la construcción del enemigo. Reconocen al diferente, pero, elaboran su representación e interpretan su existencia hasta hacer de él un enemigo al que se le ofrece la disyuntiva del sometimiento o la desaparición". ${ }^{29}$

Una mirada laudatoria del fenómeno de la tradición jurídica española implica trasladar al tiempo presente criterios de legitimidad relativos al orden social y jurídico, que pugnan con los grandes desafíos que abordamos como humanidad. Necesitamos estudiar el derecho español y el precolombino no sólo por el interés que suscitan sus instituciones y derecho. También, porque aspiramos a conocer cómo se han ido formando las limitaciones históricas para el desarrollo de una cultura democrática y de derechos humanos en los tiempos actuales. -Aunque cabe recordar que, en el mundo indígena prehispánico, también encontramos que hubo estratificación social y jerarquías fundadas en la violencia-. La debilidad que presenta la sociedad iberoamericana en esta materia puede tener como una de sus causas, la cultura jurídica. El derecho ha contribuido a recrear la mentalidad patriarcal. ${ }^{30}$

\footnotetext{
${ }^{28}$ Thomas Duve, "Los desafíos de la Historia Juridica Europea", Anuario de Historia del Derecho Español, tomo 86, 2016, p. 831.

${ }^{29}$ Villacañas Berlanga, José, La formación de los reinos hispánicos, España, Espasa, 2006, p. 25.

${ }^{30}$ Valga precisar que, sin embargo, ello no ha impedido la formación de propuestas teóricas o doctrinales que promuevan la dignidad humana y los derechos humanos.
} 
Una didáctica que proponga el surgimiento del estudiante como sujeto histórico amoroso no puede agotarse en la visión positiva de la expansión del derecho romano-católico. Esta didáctica debe dar a conocer cómo estos fenómenos contribuyeron a formar una determinada cultura jurídica, donde el orden político, social, económico y cultural admite la violencia, la dominación, la discriminación y la consecuente jerarquía social, todo lo cual genera "hábitos patriarcales".

\section{Enseñanza del derecho precolombino, pluralismo jurídico e interculturalidad}

El reconocimiento constitucional de los pueblos indígenas plantea un enorme desafío a la historia del derecho en sus tres dimensiones: cátedra, metodología de investigación y objeto de estudio. Es un hecho que los programas tradicionales están al servicio de una visión eurocentrista, lo cual implica no preparar a los futuros abogados para administrar el pluralismo jurídico y la interculturalidad.

La emergencia de lo indígena como fenómeno intrascendente en el contexto de la tradición jurídica occidental ha tenido como consecuencia que sea más relevante el reconocimiento del derecho indígena y su caducidad, en vez de la historia de las comunidades indígenas y su enfrentamiento con el derecho del colonizador. En vez de caracterizar la diversidad precolombina, los indianistas se ocupan de hablar de la regulación europea de esta diversidad. Las comunidades emergen ante los estudiantes carentes de historia, y lo hacen sin identidad propia, en un conjunto genérico englobado bajo la denominación de derecho indígena precolombino.

La aceptación del derecho consuetudinario indígena por la Monarquía española se hizo en un contexto de civilización y evangelización, es decir, de transformación cultural de lo autóctono. Es un típico caso de multiculturalismo y no de interculturalidad. ${ }^{31}$

Una adecuada comprensión de este fenómeno requiere que se conozca la historia de lo que se transformó. Esta historia da noticia de la convivencia a lo largo de varios siglos. Por un lado, se encuentran ordenamientos jurídicos no respaldados en un andamiaje estatal y basados en la fuente formal del derecho costumbre jurídica, y, por el otro, ordenamientos emanados del poder político estatal, fundados en la fuente formal ley. ${ }^{32}$

\footnotetext{
${ }^{31}$ Véase por todos Giménez Romero, Carlos, "Pluralismo, Multiculturalismo e Interculturalidad", Educación y futuro: revista de investigación aplicada y experiencias educativas, núm. 8, pp. 11-20.

${ }^{32}$ Véase Palacios Romero, Francisco, "De objeto colectivo a sujeto colectivo: paradigmas históricos de cultura constitucional y premisas de legitimación del derecho indigena", lus Fugit, núm. 19, pp. 265-300.
} 
El reconocimiento de la interculturalidad implica darle a la diversidad un sentido transformador y liberador respecto de instituciones tradicionales de dominación de lo diverso. En síntesis, para la historia del derecho, el desafío del pluralismo jurídico y de la interculturalidad implica, en lo metodológico, un reforzamiento de los lazos con la antropología y la arqueología; en lo tocante al objeto de estudio, una ampliación del pasado jurídico que resulta de interés para la formación de los estudiantes; $y$, respecto de la cátedra, una ampliación de sus contenidos - estudio de las sociedades precolombinas- y un reforzamiento de una didáctica destinada a promover la conciencia crítica.

La protección actual de la concurrencia legítima de diversidad de identidades, en un mismo medio social, pugna con la mentalidad patriarcal tradicional, que organiza dicha concurrencia en términos de superioridad-inferioridad. Por ende, la historia del derecho, como cátedra y fuente de investigación, tiene la tarea de denunciar las raíces de dicha mentalidad y su protección y promoción por la normativa jurídica.

\section{Conclusiones}

Una didáctica de la historia del derecho centrada en el aprendizaje puede contribuir a la identificación de la zona oscura de la tradición jurídica occidental y, en esa medida, a superar los hábitos patriarcales amparados por el derecho a lo largo de la historia de Occidente, en los últimos 2500 años. Ello implica adoptar un modelo pedagógico sociocrítico y exponer a los estudiantes a un proceso formativo como abogados promotores de una nueva cultura jurídica fundada en la democracia, los derechos humanos, el pluralismo jurídico y la interculturalidad. Se trata de formar abogados que actúen como sujetos históricos amorosos y que, en esa medida, denuncien los hábitos patriarcales.

Las nuevas concepciones sobre el aprendizaje y la contribución de la universidad a la formación profesional deben abordar el problema más relevante al que se enfrenta el profesional del derecho en el siglo xxI, como es la consolidación de la sociedad de la interculturalidad, del pluralismo jurídico, de la democracia y los derechos humanos. Este nuevo modelo de organización social implica una valoración de quienes, a lo largo de la historia, han sido objeto de una violencia que se presenta como legítima. Esta valoración es fundamental pues, en la cátedra, se adopta una posición de aparente neutralidad respecto a tal violencia.

La tradición jurídica occidental, y su cultura más antigua, rescató en la narración y el objeto de estudio un conjunto de hábitos que ampararon relaciones personales fundadas en la violencia. De ahí, resultó dominación, discriminación 
y una jerarquía social que favorece a los varones. Valores del pasado vienen siendo trasmitidos hasta el presente como meros objetos contenidos en una narración, sin reparar en que la aparente neutralidad de la enseñanza ante la explicación binaria libre/esclavo, capaz/incapaz contiene un modelo social que todavía perdura en muchos espacios sociales. Este mismo se erige como obstáculo para la consolidación de la nueva cultura jurídica de la democracia y los derechos humanos: una cultura humanitaria.

La historia del derecho, vista como cátedra, investigación y acontecer, puede hacer una contribución decisiva a la formación de los abogados que requerirá esta nueva sociedad que se viene consolidando en nuestro suelo iberoamericano. Desde el terreno de la didáctica de la historia del derecho, el diseño de un plan de formación de la conciencia crítica con base en un fenómeno histórico, como es la denominada polémica de Indias, permite desarrollar habilidades cognitivas y procedimentales, así como actitudes en el plano político normativo, al conectando pasado y presente de manera fluida. Finalmente, estas habilidades son una contribución decisiva en la formación del jurista llamado a cumplir un rol activo en la consolidación de la nueva cultura jurídica del siglo XxI.

\section{Bibliografía}

Bascuñán, Aníbal, Pedagogía Jurídica, Chile, Editorial Jurídica, 1954.

Caroni, P10, La Soledad del Historiador del Derecho. Apuntes sobre la conveniencia de una disciplina diferente, Madrid, Universidad Carlos 111 de Madrid, 2010.

De Arvizu y Galarraga, Fernando, "La enseñanza de historia del Derecho: reflexiones en busca de una polémica “, Anuario de Historia del Derecho Español, 1988.

Duve, Thomas, "Los desafíos de la Historia Jurídica Europea”, Anuario de Historia del Derecho Español, tomo 86, 2016.

Elgueta Rosas, María Francisca y Palma González, Eric Eduardo, "Una propuesta de clasificación de la clase magistral impartida en la Facultad de Derecho", Revista Chilena de Derecho, vol. 41, núm. 3. [Consulta: 1 de junio, 2017]. Disponible en: http:// www.scielo.cl/scielo.php?pid=S0718-34372014000300006Ctscript=sci_abstract

Espinosa Gallegos-Anda, Carlos y Caicedo Tapia, Danilo, Derechos Ancestrales. Justicia en Contextos Plurinacionales, Ecuador, Ministerio de Justicia y Derechos Humanos, 2009. Freire, Paulo, Pedagogía del Oprimido, México, Siglo XXI.

GimÉnez Romero, Carlos, "pluralismo, multiculturalismo e interculturalidad”, Educación y futuro: revista de investigación aplicada y experiencias educativas, núm. 8.

GonZÁLEZ BALLESTEROS, IvÁN, "El estereotipo del bárbaro y la imagen de la civilización en el occidente romano en la Geografía de Estrabón”, Espacio, Tiempo y Forma, serie 2, tomo 22. 
Hawes, Gustavo, Pensamiento crítico en la formación universitaria. Documento de Trabajo 2003/6 Proyecto Mecesup TAL 0101, Universidad de Talca, 2003.

LeVaggi, Abelardo, “Consideraciones sobre la enseñanza de la Historia del Derecho”, Revista de Historia del Derecho Ricardo Levene, núm. 27.

Maturana, Humberto y Varela, Francisco, El árbol del conocimiento. Las bases biológicas del entendimiento humano, Editorial Universitaria, 1996.

Merryman, John Henry, La tradición Jurídica romano-canónica, México, FCE, 1971.

Morán Martín, Remedios, Materiales para un curso de Historia del Derecho, Madrid, UNED, 2010.

Palacios Romeo, Francisco, "De objeto colectivo a sujeto colectivo: paradigmas históricos de cultura constitucional y premisas de legitimación del derecho indígena”, Ius Fugit, núm. 19.

Palma González, Eric, Historia del Derecho. Tomo I. Primera Parte. La Historia del Derecho en Chile: investigación, método y cátedra, Talca, Universidad de Talca, 1995. Palma GonzÁlez, ERIc, Historia del Derecho I. Introducción, Historia del Derecho Español, Los Pueblos Prerromanos, Chile, Universidad Central de Chile, 2001.

Palma GonzÁlez, Eric, "Historia del Derecho en América Latina en el siglo XXI: el caso de Chile, Colombia y México”, Revista Ambiente Jurídico, núm. 11.

Palma GonzÁlez, Eric, "Pasado, presente y futuro de la Historia del Derecho en Chile", Revista electrónica alemana Forum Historiae Iuris. [Consulta: mayo, 2017]. Disponible en: http://www.forhistiur.de/es/2009-03-palma-gonzalez/?l=es

Palma GonzÁlez, ERIc, "Reflexiones en torno a una concepción polifacética para una historia del derecho de los siglos XIX y xx”, Ius et Praxis, año 3, núm. 2.

Palma González, Eric y Elgueta Rosas, María Francisca, "Enseñanza de la historia del derecho centrada en el aprendizaje de los estudiantes a lo largo de 115 años de la fundación de la cátedra (Chile, 1902)”, Revista Jurídica, num. 12.

RuIz Rodríguez, Ignacio, Historia del Derecho y de las Instituciones Españolas, Madrid, Dykinson, 2011.

Salvat Monguillot. Manuel, Breve historia del estudio del derecho, Chile, Instituto de Historia del Derecho Juan de Solórzano y Pereyra, 2001.

Silva C., JAIme, "Biología de la regulación emocional su impacto en la Psicología del afecto y la psicoterapia”, Terapia psicológica, vol. 21, núm. 2.

SeoAne, María IsABel, "Los recursos didácticos a utilizar en la enseñanza de la Historia del Derecho Argentino”, Revista de historia del derecho, núm. 27.

SuÁrez Blázquez, Guillermo, "La patria potestad en el derecho romano y en el derecho altomedieval visigodo", Revista de Estudios Histórico Jurídicos, núm. 36. [Consulta: mayo, 2017]. Disponible en: http://www.scielo.cl/scielo. php?pid=S0716-54552014000100005\&tscript=sci_abstract

UNESC0, "Recopilación de recomendaciones relativas a la situación del personal docente de los años 1966 y 1997”, UNESCO. [Consulta: enero, 2017]. Disponible en: http:// unesdoc.unesco.org/images/0016/001604/160495s.pdf 
Villacañas Berlanga, José, La formación de los reinos hispánicos, España, Espasa, 2006. Wolkmer, Carlos Antonio, "Pluralismo jurídico: nuevo marco emancipatorio en América Latina”, FLACSO, Documento de trabajo, editor CENEJUS, 2003. [Consulta: diciembre, 2016]. Disponible en: http://biblioteca.clacso.edu.ar/clacso/ otros/20111021100627/wolk.pdf

Zambrana, Patricia, "Metodología de la enseñanza de la Historia del Derecho ante el Espacio Europeo de Educación Superior. Técnicas docentes”, en Actas del II Congreso de Innovación Docente en Ciencias Jurídicas [Recurso electrónico] / coord. por Juan Manuel Ayllón Díaz-González, 2009;

ZAmbranA, PATRicia, "La adquisición de competencias en el marco de la enseñanza de la Historia del Derecho: la preparación y exposición de temas por los alumnos como técnica docente”, Revista Jurídica de Investigación e Innovación Educativa, núm. 1. Zambrana, Patricia, Salamanca, escuela de juristas. Estudios sobre la enseñanza del Derecho en el Antiguo Régimen, Madrid, Universidad Carlos III de Madrid Dykinson, 2012.

ZAMBrAnA, PATRICIA, “Las enseñanzas histórico-jurídicas y romanísticas en las principales facultades de derecho de los Estados Unidos de América” Revista de Estudios Histórico Jurídicos, núm. 26. 